\title{
Le pouvoir et les Beaux-Arts sous la Restauration
} (1815-1830)

Thèse de doctorat en histoire sous la direction de Francis Démier, Université Paris 10-Nanterre, soutenue le 18 juin 2002 devant un jury constitué de Jean-Pierre Chaline (président), Marie-Claude Chaudonneret, Jean-Paul Clément, Francis Démier et Ségolène Le Men.

\section{Émeline Wirty}

\section{OpenEdition}

Journals

Édition électronique

URL : http://journals.openedition.org/rh19/457

DOI : $10.4000 /$ rh19.457

ISSN : $1777-5329$

Éditeur

La Société de 1848

Édition imprimée

Date de publication : 1 décembre 2002

Pagination : 284-289

ISSN : 1265-1354

Référence électronique

Émeline Wirty, "Le pouvoir et les Beaux-Arts sous la Restauration (1815-1830) », Revue d'histoire du XIXe siècle [En ligne], 25 | 2002, mis en ligne le 20 juin 2005, consulté le 10 décembre 2020. URL : http:// journals.openedition.org/rh19/457 ; DOI : https://doi.org/10.4000/rh19.457

Ce document a été généré automatiquement le 10 décembre 2020.

Tous droits réservés 


\section{Le pouvoir et les Beaux-Arts sous la Restauration (1815-1830)}

Thèse de doctorat en histoire sous la direction de Francis Démier, Université Paris 10-Nanterre, soutenue le 18 juin 2002 devant un jury constitué de Jean-Pierre Chaline (président), Marie-Claude Chaudonneret, Jean-Paul Clément, Francis Démier et Ségolène Le Men.

\section{Émeline Wirty}

Après l'épisode révolutionnaire, la société entame sa reconstruction et ce, dès l'Empire. En 1814, l'appareil d'État français est toujours napoléonien. Dans la Déclaration de SaintOuen (2 mai 1814), Louis XVIII affirme ne pas avoir l'intention de tout remettre en cause. Ainsi, l'État napoléonien fonctionne encore très bien sous la Restauration. L'État, pendant ces quinze années, reste napoléonien. Et on espère qu'avec le retour des Bourbons, un cadre propice à faire enfin aboutir des mesures freinées par un contexte de guerre pourrait se dessiner. Selon nous, la monarchie restaurée n'est alors pas du tout perçue comme un handicap. Depuis la Révolution, et encore plus après le despotisme militaire dont s'est rendu coupable l'Empereur, on est en quête d'une vraie stabilité. La Restauration est alors justement vue comme son possible berceau. Et en matière de Beaux-Arts, le pouvoir (qu'il s'agisse des monarques ou bien d'administrateurs) va savoir adapter la majeure partie des grandes institutions artistiques du moment, à la nouvelle donne politique, économique et sociale qui caractérise la France de la Restauration. Nous avions le sentiment, au commencement de cette recherche, que la Restauration était plus féconde et novatrice qu'on voulait bien le laisser encore parfois penser. Par conséquent, nous avons fait le choix de nous limiter strictement à ces quinze années, de manière à considérer pleinement leurs apports, tout en sachant que la chronologie de l'histoire n'est pas un choix qui pourrait laisser supposer l'existence d'une quelconque coupure. Cette dernière serait, en effet, bien artificielle car nombre d'administrateurs et d'institutions survivent aux changements de régime. Et globalement, les Beaux-Arts ne calquent pas forcément ou systématiquement leur marche sur celle de l'Histoire. De manière générale, les travaux qui existent ont tendance à valoriser les artistes. L'étude du rapport État/artistes, pour sa part, a en grande partie privilégié la III ${ }^{e}$ République, 
présentée comme ayant été la plus préoccupée de la puissance publique dans la culture. Quant à la perspective sur le XIX ${ }^{\mathrm{e}}$ siècle, elle a tendance à occulter la monarchie et ce, à tort, car la politique culturelle fait partie intégrante de ses grandes préoccupations. Pour savoir si le cadre monarchique avait été propice aux Beaux-Arts, cela impliquait, selon nous, d'adopter une approche double, à la fois institutionnelle et quantitative (comptable).

L'approche institutionnelle

Tout l'enjeu a été de mesurer, à travers les raisons qui ont présidé aux remaniements successifs de l'administration générale des Beaux-Arts, l'importance donnée à ces derniers. Ceci a impliqué d'étudier minutieusement la vocation et l'organisation de chaque institution de tutelle. Ceci a impliqué aussi de mesurer ce que le régime des Bourbons avait gardé ou rejeté des grandes institutions artistiques dont il avait héritées, ce qui est revenu à déceler les continuités et les ruptures avec l'Ancien Régime mais aussi avec la période révolutionnaire et impériale, une démarche qui a consisté à quantifier leur poids respectif à travers l'appareil administratif, intermédiaire entre le roi et les artistes, appareil administratif qui se trouve partagé entre la Maison du roi, le ministère de l'Intérieur et la préfecture de la Seine. Et au sein de cette administration centralisée, plusieurs grandes institutions ont joué un rôle capital : les musées, l'Institut, l'Académie de France à Rome, les écoles d'art, les manufactures. Nous avons donc choisi d'articuler notre réflexion autour de ces institutions artistiques majeures, de façon, en multipliant ainsi les angles d'accroche, à élargir notre vision d'ensemble, même si nous nous sommes limités, dans le cadre de cette recherche, aux institutions proprement parisiennes, un choix motivé aussi par le caractère centralisateur de l'administration sous la Restauration.

L'approche quantitative

L'artiste, sous la Restauration, joue un rôle social de tout premier plan. Comme sous l'Empire, il est appelé pour les fêtes et cérémonies commémoratives du régime. La légitimité politique passe aussi par les arts. Les artistes servent, d'une certaine manière, à asseoir l'autorité du régime en place, à rebâtir une image nationale de la monarchie et ce, au travers de programmes politiques qui leur sont confiés. La volonté de réconciliation nationale, de reconstruction sociale, est très présente et les artistes sont ainsi conviés à retisser ce que l'on peut appeler un lien social. La culture (dont les Beaux-Arts sont une composante) est un enjeu national et les dépenses d'argent qui vont avec revêtent un caractère indéniablement utilitaire. Alors, tout l'enjeu a été de pouvoir mesurer les conséquences réelles sur le plan social et financier de cette accession de l'artiste à de véritables responsabilités nationales. Si l'évolution de son statut se mesure à l'intérêt porté par le pouvoir aux institutions artistiques majeures, elle se dessine également, selon nous, au regard des différents soutiens financiers ou marques de reconnaissance dispensés par le régime en place.

Pour définir de manière neuve (c'est en effet assez peu fait sur la longue durée) la politique de l'État en termes comptables et mesurer avec exactitude le poids des BeauxArts dans une période de restriction budgétaire, nous avons, à chaque fois que les archives le permettaient, procédé à la reconstitution de la ligne budgétaire de chaque établissement artistique majeur. Cette entreprise s'est révélée difficile par son ampleur d'une part, par le fait aussi que sous la Restauration, les budgets ne sont pas annuels mais mensuels. Les budgets dévolus aux Beaux-Arts constituent un indicateur précieux, les différents types de soutiens, mis en place, maintenus, voire améliorés, par la Restauration 
et regroupant retraites, pensions, secours, logements, indemnités, encouragements et gratifications, en constituent un autre.

Enfin, une étude des distinctions honorifiques dispensées aux artistes ainsi qu'aux administrateurs d'établissements artistiques, renseigne également sur la façon dont ils ont été récompensés par le régime des Bourbons. Ainsi, être décoré de l'Ordre de SaintMichel ou de celui de la Légion d'honneur constituent autant de témoignages de l'intérêt porté aux artistes ou aux administrateurs par le régime. Nous avons donc ambitionné, à l'aide principalement des dictionnaires biographiques de l'époque, de l'Almanach royal et des archives (pour la plupart inédites) conservées au Musée de la Légion d'honneur et des Ordres de chevalerie, d'en dresser un recensement quasi exhaustif.

Notre travail s'est articulé autour de trois axes majeurs de recherche, de réflexion En premier lieu, il s'avère que l'administration des Beaux-Arts sous la Restauration est encore marquée, à plusieurs égards, de l'empreinte napoléonienne, une continuité par rapport à l'État napoléonien qui s'intègre du reste dans une politique de récupération plus générale de l'héritage impérial. On a ainsi une administration à la fois fortement centralisée et tenue à l'écart, autant qu'il peut être permis de l'envisager, des aléas de la conjoncture politique. Les Bourbons n'ont donc pas voulu, à leur retour, effacer cet aspect du legs impérial. Une raison, plutôt pragmatique, émerge. L'outil remarquable qu'est l'État centralisé et moderne, laissé par Napoléon, constitue une véritable chance. Le très perspicace Louis XVIII l'a bien compris. Ce système au combien efficace n'a donc pas été transformé en profondeur. Ainsi, le retour des Bourbons n'a pas engendré de ruptures immédiates.

Cet état d'esprit se traduit alors par une stabilisation, voire une consolidation et une amélioration, réponse administrative à la volonté politique, des principales institutions artistiques (institutions de tutelle comme établissements) tout au long de la Restauration. On assiste à un réel accroissement de la fonction administrative au sein des Beaux-Arts. Par exemple, la création, au sein de la Maison du roi, d'un département des Beaux-Arts, en 1824 , constitue une étape fondamentale. La réunion, en son sein, des principales institutions artistiques, des instances présidant aux cérémonies, de la liste civile et de la caisse de vétérance lui donne des allures de ministère. Sa vocation est, du reste, ministérielle et ce, même si son existence légale se borne à la Maison du roi. À ce moment précis, nous pensons qu'il peut être considéré à juste titre comme un ministère des Beaux-Arts embryonnaire. Il porte ainsi en lui les germes du ministère des Beaux-Arts créé par le décret du 2 janvier 1870. L'organigramme de l'administration des Beaux-Arts connaît ainsi de réelles continuités. Les deux rois (et notamment Louis XVIII) ont fait preuve d'une capacité d'adaptation certaine en faisant autant appel à d'anciens serviteurs de l'Empire qu'à des hommes qui avaient démontré, par le passé, leur fidélité inconditionnelle aux Bourbons. La nomination ou la confirmation à de nombreux postes de personnalités qui occupaient, déjà sous l'Empire, parfois avant, des fonctions souvent très proches de celles occupées sous les Bourbons, sont vraiment frappantes. Les administrateurs, déjà en place, n'ont pas été systématiquement écartés, sous prétexte de services passés rendus à l'Empire. Les bureaux comme les administrations propres à chaque établissement ne souffrent pas de bouleversements de fonds au retour des Bourbons. Il semble bien que la Restauration, tout en contrôlant de manière clairvoyante la situation, a eu l'immense sagesse de laisser aller les choses simplement. De nombreux administrateurs, auxquels une vraie latitude a été laissée, ont pu, dans le cadre propice offert par la monarchie restaurée, poursuivre leur gestion de façon cohérente et assez libre. Comme sous l'Empire, les véritables choix se font dans les bureaux. C'est ainsi que 
les établissements, dont aucun est né sous la Restauration, qu'ils avaient en charge, ont connu une transition en douceur, voire une continuité, avec le régime précédent et ont ainsi pu perdurer. Et si une bonne partie de ces hommes sont des hommes d'Ancien Régime, nombreux sont ceux qui ont su s'acclimater, retrouver une place, dans une société française qui avait commencé à se reconstruire dès l'Empire. Avancer un tel état de fait, nous en avons conscience, c'est aller contre beaucoup d'idées reçues qui ont présenté la Restauration comme sclérosée, étouffante. Son administration, a fortiori celle des Beaux-Arts, parce qu'elle était placée (en grande partie) sous la tutelle de la Maison du roi, ne pouvait alors être composée que de "ministres-domestiques". Au vu, entre autres, de la lecture et de l'analyse de la correspondance administrative, cette image est bien dépassée tout comme celle d'ailleurs qui présente encore trop souvent et à tort les Beaux-Arts de la période comme dirigés par le prince et au seul service du régime. Pire, elle est inexacte et évidemment injuste envers beaucoup d'hommes qui ont trouvé passion à servir les établissements dont ils avaient la charge.

En second lieu, le maintien, voire l'augmentation, tant des sommes allouées aux institutions artistiques que des soutiens financiers destinés aux artistes constitue une autre étape. La plupart des institutions se voient, en effet, attribuer des montants très proches de ceux en vigueur sous l'Empire. D'autres bénéficient même de sommes plus élevées. Et même si les établissements relevant de la Maison du Roi sont généralement mieux lotis que ceux relevant du ministère de l'Intérieur, même si les efforts budgétaires semblent concentrés sur Paris, les Bourbons ont globalement fait bénéficier les BeauxArts d'une réelle continuité budgétaire (la même continuité est d'ailleurs observable dans le domaine salarial), ce qui était loin de constituer une évidence compte tenu de la situation financièrement précaire de la France. Cette volonté de pallier (via les différentes aides offertes aux artistes), autant que possible, les difficultés financières qui accompagnent souvent la création, est bien une façon de reconnaître les spécificités inhérentes à la profession d'artiste et par là même, les spécificités d'un groupe social. $A$ priori, aucune personne, sous prétexte qu'elle ait travaillé sous l'Empire, se trouve écartée du système de retraites, de secours ou de gratifications, qui hélas, d'abord pensé pour la Maison du roi, possède ses propres limites. Heureusement, pour les plus démunis, un ensemble d'aides, "soulagement à la misère, triste compagne du talent", constitué de pensions, vient atténuer, en partie, ce déséquilibre. L'octroi d'un logement ou, le cas échéant, d'une indemnité, là encore héritage impérial, constitue une aide des plus appréciables pour les artistes ou fonctionnaires qui en bénéficient. Les encouragements, legs de la Révolution, représentent également un apport financier précieux pour les plus méritoires. Ce panel d'aides, dont a disposé le régime des Bourbons, a donc été conservé avec sagesse (quand l'origine est antérieure à la Restauration) et amélioré, avec intelligence. Et l'attribution massive de distinctions honorifiques prestigieuses (Ordres de Saint-Michel et de la Légion d'honneur, création impériale que les Bourbons ont su adapter à leurs propres visées) tant à des artistes qu'à des administrateurs, représentatifs (et c'est capital) de toutes les institutions artistiques majeures, n'a pu que contribuer à renforcer la cohésion d'un groupe social émergent. Si le premier Ordre est officiellement destiné aux artistes, hommes de lettres ou de sciences, le second récompense les artistes bien plus que l'Empire ne l'a jamais fait.

Toutefois, et c'est notre troisième axe de réflexion, à côté de cet élan, de ce dynamisme, de cette attitude moderne, se réclamant très souvent du modèle louis-quatorzien et colbertiste, moteur en son temps de la centralisation culturelle (le règne de Louis XVI n'est en effet jamais érigé en modèle), attitude adoptée par l'État en faveur des Beaux- 
Arts et qui va bien au-delà du rôle traditionnel de protecteur des artistes, il subsiste çà et là des réminiscences d'Ancien Régime, des archaïsmes au sein du paysage artistique de la Restauration. On les trouve parfois dans le fonctionnement même des institutions. On les trouve aussi dans les motivations préexistantes, par exemple, à l'utilisation des manufactures, même si les conditions de travail des employés de ces établissements ont globalement été améliorées, pour mettre en scène le pouvoir (au travers, par exemple, de cérémonies commémoratives) et le servir. Créations de Henri II (la Monnaie des Médailles), Henri IV (la manufacture de la Savonnerie), Louis XIV (les manufactures des Gobelins et de Beauvais) ou encore Louis XV (la manufacture de Sèvres), les manufactures occupent une place un peu à part dans le paysage artistique de la Restauration, dans la mesure où elles rappellent plus que toute autre institution, via leur origine et certaines de leurs attributions (les décors éphémères accompagnant certaines cérémonies commémoratives, les cadeaux officiels, la commémoration des principaux événements du règne), les monarques d'Ancien Régime. Alliées séculaires du pouvoir monarchique, ce n'est pas la Restauration qui sera revenue sur cette prérogative. L'implication idéologique présente au sein de la politique culturelle apparaît tout aussi forte que sous l'Empire. La politique culturelle fait alors figure d'illustration idéale des vertus du régime en place. On a donc bien, d'une certaine manière, une politique en faveur des arts qui est vue comme une des composantes privilégiées du pouvoir et de sa représentation.

Les Beaux-Arts sont considérés comme un allié incontournable du pouvoir, dans la mesure où la variété des supports offerts par ceux-ci multiplient les impacts espérés par un régime en quête d'une nouvelle image. Si les actes commémoratifs, souvent imprégnés d'une connotation expiatoire, réapparaissent au retour des Bourbons, tels un spectre de l'Ancien Régime ; si ceux-ci se sont également empressés de faire disparaître des multiples supports artistiques tous les signes les plus visibles évoquant l'Empire, la monarchie restaurée, Janus aux deux visages, a aussi eu le génie de puiser dans l'histoire passée, des épisodes (présentés, grâce aux Beaux-Arts, de manière pédagogique) au pouvoir rassembleur, potentiellement capables de fédérer deux France (celle de l'Ancien Régime et celle de la Révolution).

La Restauration est bien, dans le domaine des Beaux-Arts et de leur administration, une période très féconde car il n'y a pas de blocage sur les institutions antérieures ni fixation sur un régime établi. En matière de politique économique et sociale, de vrais efforts ont été, là aussi, menés pour assurer aux Beaux-Arts, à la fois une transition en douceur avec le régime précédent et une amélioration du quotidien des artistes, encore fortement précaire pour une majorité d'entr'eux. C'est donc une vraie politique, empreinte de sagesse, qui a guidé un régime auquel rien ne parait contradictoire : puiser dans l'épisode impérial, sans pour autant devenir une pâle imitation de ce modèle, quand cela semble être porteur de promesses en matière d'efficacité, notamment dans le domaine administratif, tout en n'hésitant pas parfois à sacrifier au style "Ancien Régime". Combiner les références au modèle tant louis-quatorzien que napoléonien montre une Restauration qui a su finalement manier l'art de la synthèse avec un certain brio. Néanmoins, il y a eu échec. Comme (par exemple) la politique économique, la politique culturelle n'a pas sauvé la Restauration. Elle n'a pas constitué un élément fort dans la monarchie au moment où celle-ci entre en crise. Les institutions ont finalement échoué dans la remise en place des Bourbons et les limites de la politique artistique sont évidentes dans le projet plus vaste de restauration de ces derniers. 
INDEX

Mots-clés : 2002 